\title{
Le “Aule Verdi” per Educare all’Ambiente e Intercultura: Uno Studio di Caso
}

\author{
By
}

\section{${ }^{1}$ Gabriella Calvano (PhD Student)}

«Nella libertà dei semi vivono la speranza

e la possibilità di un mondo migliore»

(Vandana Shiva, Storia dei semi, Feltrinelli, Milano 2013)

\begin{abstract}
Environment is not only the ensemble of elements that is present in a context, but it is also the ensemble of relationships between these elements. For this reason, in the complexity's society, educate to environment entails to pay attention to people, to relationships and to dialogue opportunities among them.

This case study underlines intercultural dialogue opportunities that could be created by an educational path of environmental education in a Primary School. This experimental research has lead to the construction of a Mediterranean Garden in outward space of school. Through education to environment and biodiversity it is possible to put foundations for dialogue between different cultures, as different are biological species that form Mediterranean Garden.
\end{abstract}

Keywords: educazione ambientale, educazione interculturale, aula verde, laboratorio, biodiversità

\section{Introduzione}

L'attenzione all'ambiente e alle questioni ad esso legate è oggi più che mai avvertita come risposta ai cambiamenti climatici, all'emergenza dell'inquinamento, ma anche come conseguenza dei profondi mutamenti e della rottura degli equilibri sociali, economici, politici ed antropologici propri della realtà contemporanea.

Nella sua accezione più ampia e generica, l'ambiente si sostanzia delle relazioni e delle occasioni di comunicazione che si generano tra gli elementi che lo compongono: applicando all'ambito educativo questa definizione, le possibilità di integrazione e convivenza di ogni persona all'interno del proprio gruppo sociale crescono enormemente. Allo stesso modo, la trasformazione multiculturale e multietnica che sta 
caratterizzando le nostre società deve indurre a puntare l'attenzione sulle relazioni tra persone e gruppi culturali differenti, in modo da elaborare programmi e progetti che siano autenticamente interculturali.

Il primo passo da compiere per affrontare le questioni ambientali in prospettiva interculturale è accorgersi della presenza dell'ambiente, di percepirlo, osservarlo e studiarlo, di saperlo guardare con occhi nuovi e stupirsi di fronte ad esso. Il tema della biodiversità, in particolare, consente di coniugare la prospettiva dell'educazione ambientale con l'educazione interculturale in quanto "porta al riconoscimento della varietà e della diversità come "valori n sé" da promuovere e rispettare, costringendo a ripensare criticamente e profondamente alle manifestazioni di intolleranza e di incomprensione, alle persistenti azioni di discriminazione, dimenticate e represse» (BINANTI, 2012, p. 12 ). Espressione naturale di dialogo e di crescita a partire da questo, la biodiversità è considerata un vero e proprio bene culturale, la cui comprensione e sopravvivenza sono condizionate dalla presenza di conoscenze sociali condivise.

Educare ad un positivo rapporto con la natura è possibile e opportuno fin dalla scuola dell'infanzia, facendo attenzione ai diversi livelli di consapevolezza e di esperienza dell'ambiente più prossimo, per indurre a scoprire, pian piano, il vissuto degli altri a partire dalla riflessione sull'ambiente stesso. Grazie alla mediazione dell'educazione interculturale è possibile ricondurre i problemi dell'ambiente lontano all'esperienza soggettiva, promuovendo la consapevolezza delle responsabilità di ciascuno per la tutela dell'ambiente e la prevenzione dei disastri ecologici a partire dal punto di vista della propria cultura.

\section{Educazione ambientale in prospettiva interculturale: una proposta pedagogica}

Educazione ambientale ed educazione interculturale operano per la creazione di un nuovo habitus, di una nuova forma mentis, capace di prestare ascolto, di dialogare, di andare oltre i propri pregiudizi (CAMBI, 2006). Entrambe divengono sfondo a partire dal quale costruire un futuro in cui vigono un forte senso di responsabilità universale nei confronti della comunità terrestre e della consapevolezza del ruolo della singola persona per la costituzione e la preservazione di essa.

Il legame tra educazione ambientale ed educazione interculturale è stato ben illustrato da Piero Bertolini, il quale, nel Dizionario di Pedagogia e Scienze dell'Educazione, afferma che l'esperienza educativa «non può non essere interculturale. [...] è incontro di modi di essere, di visioni del mondo, di caratteristiche personali e sociali molto diverse. [...] tutta l'educazione è, in quanto mezzo di trasmissione di innovazione della cultura, relativa all'ambiente» (BERTOLINI P., 1996, p. 168). L'una e l'altra implicano movimenti di reciprocità capaci di valorizzare le minoranze, senza assolutizzarne i diritti, ma salvaguardando $\mathrm{i}$ «diritti collettivi poiché in essi risiedono tutti i principi costitutivi dell'umanità» (TRAVERSO, 2012, p. 65).

Educare all'ambiente suscita comportamenti responsabili rispetto a se stessi, agli altri e al mondo circostante. «Confrontarsi con l'ambiente e le culture necessita [...] che siano interiorizzati alcuni valori di fondo: la solidarietà come risposta all'insicurezza; il rispetto della diversità come risposta alla debolezza; l'assunzione di responsabilità come risposta 
all'incertezza; la lentezza, l'attenzione e la solitudine come risposta all'indifferenza» (p. 67). L'ambiente, spazio e fattore educativo per eccellenza, si fa mediatore di significati in vista della costruzione di un sapere sociale diffuso e conseguenza del continuo e proficuo rapporto dialettico tra le differenti culture.

L'educazione ambientale in prospettiva interculturale consente un'approssimazione positiva ai problemi, stringendo legami familiari con orizzonti progressivamente più ampi della realtà. Educazione ambientale ed educazione interculturale condividono, infatti, alcuni principi basilari: differenza, responsabilità, interdipendenza. L'una e l'altra, attraverso un cammino progressivo di conoscenza, trasformazione e tutela, possono favorire la nascita di un legame con l'ambiente, sia esso quello più prossimo o più remoto. Una qualsiasi proposta educativa sulla diversità può trarre maggiore linfa dall'educazione ambientale, la quale non solo riuscirà a far comprendere il valore più autentico e positivo del diverso, ma consentirà di individuare e scoprire la preziosità di connessioni, legami e relazioni. La biodiversità rappresenta una esemplificazione particolarmente significativa in tal senso. Per comprendere la biodiversità in chiave interculturale bisognerà prima di tutto imparare a guardarla con gli occhi dello stupore e della contemplazione, coltivando «un'educazione alla percezione e alla riflessione consapevole dell'ambiente prossimo, a partire dai livelli più immediati e meno vasti [...], per finire a livelli più complessi dell'ambiente remoto» (DE VITIS, 2012, p. 52).

Nel costruire un percorso di educazione ambientale in prospettiva interculturale è importante tenere ben presente non solo il livello di competenza cognitiva da cui si parte, ma anche il cammino affettivo, emotivo e sociale già compiuto da chi sarà implicato nel processo educativo/formativo. In questo modo è possibile costruire un itinerario educativo efficace e in grado di promuovere una riflessività aperta alle differenze, alla reciprocità, al rispetto e alla responsabilità nel rapporto con l'ambiente circostante. Favorendo la coesistenza di teoria e pratica, esperienza e riflessione sulla prima, componente emotiva e componente razionale, il laboratorio può essere considerato come occasione di ricerca per l'educazione ambientale in prospettiva interculturale e consentire la partecipazione degli studenti alla costruzione di nuova conoscenza. «E' uno spazio di "generatività" e di creatività che si auto motiva e accresce l'autostima, mentre estende ampiezza e spessore delle competenze di ciascuno, facendole interagire e confrontare con quelle degli altri» (p. 72).

Per gli obiettivi che ci si propone con questo lavoro, il laboratorio può favorire la conoscenza sia delle caratteristiche dell'ambiente in cui si vive sia dei più importanti tratti culturali dei Paesi di provenienza degli alunni stranieri che frequentano la Scuola Primaria "Riccardo Jannuzzi" di Andria.

Il contatto diretto con il territorio favorisce lo stupore cognitivo ed emotivo, suscita interesse per un ambiente diverso da quello che quotidianamente si frequenta, sviluppa atteggiamenti esplorativi e la capacità di affrontare e risolvere problemi; promuove l'apprendimento significativo in un clima di libertà. I progetti di educazione ambientale in prospettiva interculturale dovrebbero essere promossi e favoriti nella scuola: non prevedono, infatti, l'insegnamento/apprendimento di nuovi argomenti e conoscenze, ma spingono ad organizzare in maniera nuova i curricula, a porre maggiore attenzione sia alle scelte metodologiche che si fanno sia alle mete educative che ci si prefigge. 


\section{L'aula verde per l'educazione ambientale in prospettiva interculturale}

I giardini scolastici possono assumere grande importanza per educare all'ambiente, soprattutto nei contesti urbani, dove si assiste a una sempre minore disponibilità di spazi e tempi per poter fare esperienze a contatto con la natura. Una proposta educativa che può portare a risultati efficaci in termini di coscientizzazione degli studenti è quella di trasformare questi giardini scolastici, attraverso l'implementazione di percorsi laboratoriali, in aule verdi.

La definizione di queste strutture è contenuta nel loro stesso nome: "aula" perché si riferisce al luogo deputato all'apprendimento e alla conoscenza; "verde" perché l'obiettivo fondamentale della sua creazione è quello di introdurre nella scuola un lembo di natura. Un'aula verde serve a comprendere come funzionano gli ecosistemi e a scoprire il valore della diversità biologica, a migliorare la qualità di vita della scuola, ad imparare a gestire lo spazio e il tempo, a rispettare i ritmi della natura. Può diventare il luogo di creazione della conoscenza, anche attraverso la messa in discussione di culture diverse, non solo nello spazio (data la partecipazione, nello studio di caso che qui si presenta, di allievi di nazionalità diverse), ma anche nel tempo, poiché trattasi di progetto che coinvolge, grazie alle famiglie degli alunni, cittadini di età diverse.

L'aula verde può diventare occasione di formazione continua per docenti e alunni: è spazio di ricerca e terreno di relazione, in cui sono favoriti il confronto e la messa in discussione di convinzioni e punti di vista; è spazio di ricerca che non si esaurisce nella costruzione del giardino, ma che continua con la sua cura e tutela nel tempo.

Nella scelta dell'ambiente da riprodurre è fondamentale un'indagine preliminare sulle caratteristiche della zona in cui la scuola è situata, così da mettere l'aula verde in sintonia con il territorio circostante. In questo progetto di ricerca, la realizzazione dell'aula verde è stata preceduta dallo studio delle caratteristiche del giardino mediterraneo e dalla conoscenza, attraverso un percorso plurisensoriale, delle principali piante aromatiche che lo costituiscono. Il progetto non si è sicuramente concluso con la messa a punto dell'aula ma, al contrario, ha preso il via proprio da questo momento.

\section{Contesto di riferimento}

Il contesto territoriale nel quale il progetto si inserisce è la Scuola Primaria "Riccardo Jannuzzi" di Andria, una città collocata a sud dell'Italia, luogo simbolo della flora mediterranea e dei suoi giardini. La scuola mostra da sempre una attenzione particolare alle questioni dell'ambiente e della sostenibilità, tanto da essere l'unica realtà cittadina a promuovere iniziative, in collaborazione con UNESCO, per l'educazione allo sviluppo sostenibile.

In seguito alla maggiore presenza di immigrati, la popolazione scolastica ha visto aumentare negli ultimi anni il numero dei bambini di origine straniera e il registrarsi di episodi di mancata accoglienza di questi da parte dei compagni di classe. 
Le docenti, dopo aver esposto il problema al ricercatore, hanno con questi elaborato un progetto di ricerca con l'intento di educare gli alunni a scoprire la diversità come ricchezza, attraverso l'implementazione di un percorso di educazione all'ambiente e alla biodiversità. Nel progetto sono state coinvolte anche le famiglie dei bambini, in modo da favorire la costruzione di legami anche tra gli adulti per una maggiore integrazione non solo degli allievi ma anche delle famiglie nella comunità.

Data la presenza di ampi spazi verdi attorno alla scuola e alla presenza di un piccolo vigneto e di un piccolo uliveto nel recinto scolastico, da sempre tra le piante simbolo della macchia mediterranea, si è pensato di arricchire gli spazi con la creazione di un giardino mediterraneo, in particolare costruendo un'area dedicata alle piante aromatiche: alloro, mirto, rosmarino, timo, ginepro, lavanda.

\section{Obiettivi}

Accanto agli obiettivi di apprendimento legati agli insegnamenti disciplinari che intervengono nel corso del processo formativo, il progetto, attualmente in corso, si propone i seguenti obiettivi formativi:

- $\quad$ Favorire il contatto con gli elementi naturali di un ecosistema, avvicinandoli alla realtà scolastica.

- $\quad$ Progettare un'aula verde in armonia con il proprio territorio.

- Promuovere la partecipazione attiva degli alunni e delle rispettive famiglie all'intero progetto.

- $\quad$ Comprendere lì importanza del rispetto degli impegni presi, delle regole e dei compagni.

- $\quad$ Promuovere la conoscenza e il rispetto delle culture altre.

- $\quad$ Favorire la consapevolezza della ricchezza nella diversità.

- Incentivare la collaborazione ed il lavoro di gruppo.

\section{Destinatari}

Il progetto ha coinvolto gli alunni di due classi quarte e di due classi quinte della Scuola Primaria "Riccardo Jannuzzi" di Andria, per un totale di ottanta alunni, e le rispettive famiglie. In ciascuna classe impegnata nel progetto sono presenti due o tre bambini di nazionalità diversa, per un totale di undici alunni stranieri.

\section{Tempi e spazi}

Azione 1 - Analisi del contesto e dei bisogni; stesura del progetto (settembre 2013). Aqione 2 - Attivazione del laboratorio di lettura animata della fiaba "Camilla e il mondo dei giardini" (ottobre - novembre 2013)

Azione 3 - Progettazione dell'aula verde, preparazione e messa a punto di essa (novembre 2013 - maggio 2014).

\section{Tabella n. 1 - Cronoprogramma del progetto}




\begin{tabular}{|l|l|l|l|l|l|l|l|l|l|}
\hline Azione & Settembre & Ottobre & Novembre & Dicembre & Gennaio & Febbraio & Marzo & Aprile & Maggio \\
\hline Azione 1 & & & & & & & & & \\
\hline Azione 2 & & & & & & & & & \\
\hline Azione 3 & & & & & & & & & \\
\hline
\end{tabular}

Il progetto prevede il monitoraggio costante delle azioni, delle attività e dei risultati che di volta in volta sono raggiunti, in modo da poter eventualmente modificare quanto previsto nel caso in cui fosse necessario.

Le attività di lettura animata, a cui ha partecipato una classe per volta, sono state svolte nella biblioteca della scuola. L'aula verde è stata implementata nello spazio verde della scuola.

\section{Attività significative}

\subsection{Azione 1}

Analisi del contesto e dei bisogni e stesura del progetto formativo (8 ore).

In questa fase del lavoro sono stati coinvolti il dirigente scolastico, i docenti referenti dell'educazione ambientale e dell'educazione interculturale della scuola e gli undici docenti delle classi coinvolte nel progetto (sia gli insegnanti curriculari sia gli insegnanti di sostegno).

La scelta delle classi da coinvolgere è stata dettata sia in seguito alla registrazione, nel corso del secondo quadrimestre dell'Anno Scolastico 2012/2013, di episodi di difficoltà ad integrare bambini di provenienza diversa (acuitisi in occasione delle festività della Pasqua cristiana) sia per la maggiore sensibilità e disponibilità da parte dei docenti di classe ad impegnarsi per la risoluzione delle difficoltà incontrate.

Attraverso un questionario a risposta multipla, somministrato ai docenti coinvolti nel progetto (docenti delle classi e referenti per l'educazione ambientale e l'educazione interculturale) e al dirigente scolastico, sono stati definiti i tempi di attuazione del percorso formativo, le metodologie e gli approcci da adottare, gli obiettivi da perseguire. In particolare di sono riscontrate le seguenti risposte:

1. "Su quale aspetto credi sia importante insistere per la definizione degli obiettivi formativi? (fino a due preferenze)"

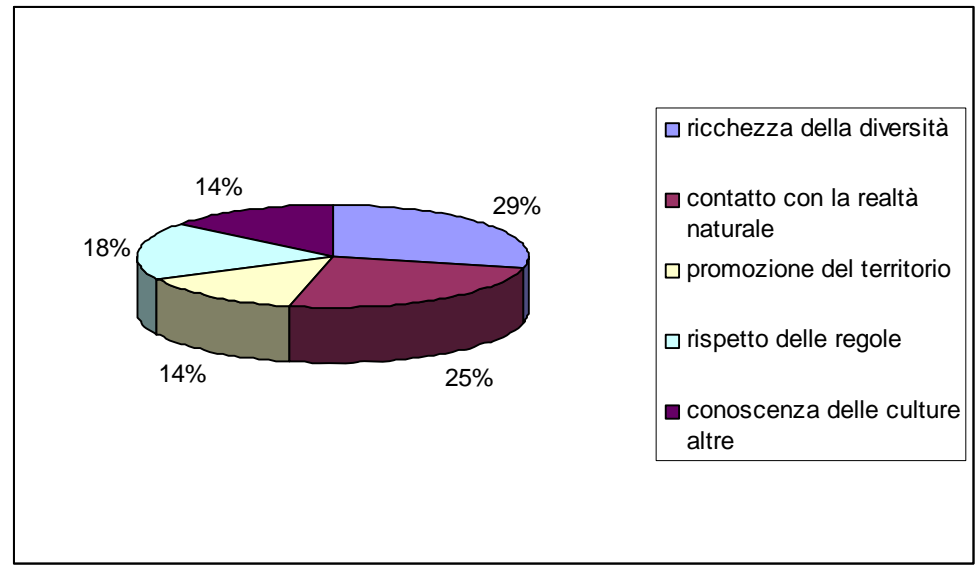

Figura n. 1 - Risposte domanda 1 
2. "Quale ritieni possa essere il metodo che consenta di raggiungere in modo più efficace gli obiettivi del progetto?"

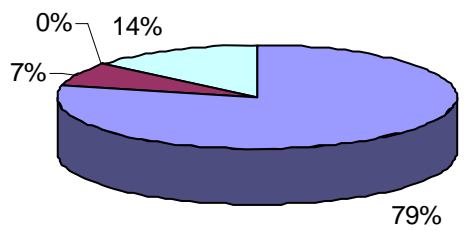

Figura n. 2 - Risposte domanda 2

Tutti i docenti e il dirigente scolastico hanno concordato che i tempi del progetto debbano estendersi all'intero anno scolastico (da ottobre a maggio), con l'intento di proseguire il progetto anche per l'anno scolastico 2014/2015 per gli alunni delle attuali classi quarte (quinte del prossimo anno scolastico) e di coinvolgere nel progetto le altre classi frequentate da allievi figli di immigrati.

\section{2. Azione 2}

Laboratorio di lettura animata della fiaba "Camilla e il mondo dei giardini" (quattro incontri per ciascuna classe da due ore ciascuno).

Per introdurre gli alunni al tema della diversità biologia, a partire dalla quale riflettere sulla diversità tra le varie culture, si è pensato di lavorare con il giardino mediterraneo, caratteristico del territorio in cui la scuola è inserita, all'interno del quale convivono, in perfetta armonia, alberi e piante aromatiche con prerogative anche molto diverse. I docenti e il ricercatore hanno deciso di guardare a questo tema servendosi del testo "Camilla e il mondo dei giardini. Una fiaba di educazione ambientale" (CICIRELLI, 2010), insistendo sulla parte della fiaba in cui vengono raccontate le avventure che la protagonista vive proprio nel giardino mediterraneo.

Nel corso del primo incontro, è stata letta la fiaba e i bambini sono stati condotti dalle insegnati alla riflessione sul testo e sui contenuti in esso presenti; successivamente gli alunni sono stati avviati alla riflessione e alla discussione grazie a delle domande-stimolo preparate dalle docenti di classe.

Il secondo appuntamento è stato così organizzato:

- $\quad$ Recupero, da parte degli alunni, dei contenuti principali della parte della fiaba raccontata nel primo incontro. 
- $\quad$ Riflessione sul tema del giardino e sulla sua importanza per l'equilibrio del pianeta, nonché sulle caratteristiche del giardino mediterraneo. La riflessione su questo è stata guidata dagli educatori del Centro di Educazione Ambientale di Ruvo di Puglia, uno dei comuni limitrofi ad Andria, che da anni implementano nella scuola "Jannuzzi" progetti di educazione ambientale. In particolare, attraverso l'utilizzo di tre dei cinque sensi (olfatto, vista e tatto), gli allievi hanno fatto esperienza, in classe, delle caratteristiche delle erbe aromatiche proprie del giardino oggetto di studio e, nello spazio verde della scuola, degli alberi di ulivo e vite presenti.

Durante il terzo incontro si è recuperato quanto fatto nell'appuntamento precedente, in modo da poter riflettere sugli aspetti del giardino mediterraneo che sono risultati essere degni di nota e significativi per gli allievi.

Nella stessa seduta è stata proposta agli alunni una seconda traccia di riflessione dal titolo "Io sono un bel giardino", grazie alla quale gli allievi hanno potuto immaginare e narrare se stessi e le loro caratteristiche e capacità (fisiche, caratteriali e relazionali) paragonandosi ad un giardino. Gli elaborati sono stati, poi, liberamente condivisi tra i piccoli studenti e si è portati loro ad acquisire consapevolezza non solo che ciascuno è, al pari del giardino mediterraneo, determinato da tratti e aspetti specifici, ma anche che ciascuno, pur nelle sue diversità, anche culturali, è bello e perfetto a suo modo.

Nel quarto ed ultimo appuntamento dell'Azione 2, dopo aver recuperato quanto di significativo emerso nell'incontro precedente, gli alunni sono stati coinvolti in un laboratorio di artistico che ha invitato loro a immaginare il proprio giardino mediterraneo scolastico e a riprodurlo, negli spazi e nella distribuzione delle erbe aromatiche, su un foglio da disegno e attraverso l'utilizzo dei semi delle erbe stesse.

\subsection{Azione 3}

La progettazione dell'aula verde, preparazione e messa a punto di essa ha previsto 3 incontri iniziali di progettazione del giardino da 2 ore ciascuno, per un totale di 8 ore, e un incontro settimanale da 2 ore ciascuno (da gennaio a maggio: 18 incontri per un totale di 36 ore) in cui ciascuna classe si recherà nel giardino per la cura e la manutenzione di esso.

L'Azione 3 è stata avviata nel mese di novembre e prevede anch'essa l'utilizzo della metodologia laboratoriale, la quale favorisce la progettazione partecipata, la didattica attiva e per competenze e l'agire riflessivo.

Nel corso di quest'azione è stato chiesto agli alunni di progettare concretamente l'aula verde, il giardino mediterraneo della propria scuola: gli studenti hanno individuato e suddiviso gli spazi verdi della scuola in modo che ciascuna porzione del giardino potesse essere opportunamente preparata per la semina delle erbe aromatiche mediterranee, nei giusti tempi, come da schema seguente.

Tabella n. 2- Tempi della semina

\begin{tabular}{|l|l|l|l|l|}
\hline & Novembre & Gennaio & Marzo & Aprile \\
\hline Alloro & & & & \\
\hline Mirto & & & & \\
\hline Rosmarino & & & & \\
\hline Timo & & & & \\
\hline
\end{tabular}




\begin{tabular}{|l|l|l|l|l|}
\hline Ginepro & & & & \\
\hline Maggiorana & & & & \\
\hline Lavanda & & & & \\
\hline
\end{tabular}

A ciascuna classe è stata affidata la cura e la manutenzione di due sezioni del giardino. La preparazione del terreno, la semina e la cura dell'aula verde, che riguarderà soprattutto il periodo compreso tra gennaio e maggio, saranno fatte soprattutto grazie all'ausilio e alla disponibilità dei nonni di alcuni alunni che renderanno concreta la partecipazione delle famiglie al progetto.

\section{Valutazione}

La complessità e la flessibilità del progetto, attualmente in corso, non consente di tracciare una proposta valutativa definitiva (valutazione che è fatta in itinere e che sarà fatta ex post): è possibile, tuttavia, individuare alcuni indicatori a partire dai quali poter esprimere delle opinioni iniziali sull'andamento del progetto.

In particolare è possibile individuare dati quantitativi e qualitativi per la valutazione.

Dati di tipo qualitativo.

I docenti, di volta in volta, potranno avere un feedback sulla positività dell'iniziativa: attraverso l'osservazione degli alunni e dei loro lavori, le impressioni dei costoro su quanto fatto, la condivisione di quanto prodotto a conclusione di ciascun appuntamento. In questo modo potranno verificare l'efficacia delle strategie utilizzate e delle metodologie adottate. I docenti hanno anche la possibilità di cogliere non solo che cosa gli allievi hanno appreso, ma anche il modo in cui ciò è avvenuto e se le competenze, stimolate nel corso delle attività, hanno suscitato negli allievi atteggiamenti positivi verso i compagni di cultura diversa, l'ambiente e il proprio territorio.

La partecipazione alla cura e alla coltivazione del giardino mediterraneo della scuola rivelerà l'adesione e il grado di soddisfazione delle famiglie rispetto al progetto.

Dati di tipo quantitativo.

A conclusione del progetto verrà somministrata (in forma specifica per alunni, docenti e dirigente scolastico e famiglie) una scala di valutazione costruita partendo dagli obiettivi che ci si era preposti ad inizio del percorso. Dalla comparazione dei dati sarà possibile dedurre se il progetto sia riuscito ad incidere su alcuni aspetti della vita delle persone coinvolte. La scala sarà strutturata in tre aree: aspetti relazionali, percezione del proprio ruolo all'interno della comunità di appartenenza, partecipazione alla vita comunitaria.

\section{Conclusioni}

In sede di conclusioni, seppur provvisorie poiché il progetto è attualmente in corso, è possibile fare alcune considerazioni.

Gli alunni stanno facendo un percorso in cui non sono privilegiati l'apprendimento logico-matematico e in cui non viene chiesto loro semplicemente di acquisire e riproporre dei contenuti: grande rilevanza stanno avendo gli aspetti emotivi e relazionali 
dell'apprendimento. Si pensi, ad esempio, alle attività di immaginazione, scrittura e messa in comune, dell'idea di se stessi o alla conoscenza delle piante attraverso il contatto sensoriale.

Le produzioni scritte e grafiche degli alunni sono i risultati tangibili di questo percorso, ma il vero risultato che sta cominciando a gratificare le docenti è la maggiore capacità di collaborazione tra gli alunni, la maggiore propensione all'ascolto dell'altro e della sua storia e cultura, nonché la grande soddisfazione che gli alunni mostrano nell'attesa e a conclusione degli appuntamenti settimanali con il progetto.

Il percorso sta offrendo agli insegnanti occasioni molteplici di riflessione e auto-analisi delle prassi attivate, consentendo di:

- rifiutando il ruolo di docente come unico depositario di saperi da trasmettere;

- valorizzando la "ricerca insieme" e la cooperazione;

- riconoscendo nelle differenze tra gli alunni valori rilevanti e significativi, che portano a forme particolari di creatività, di originalità di pensiero;

- proponendo l'immagine dell'insegnante come persona emotivamente coinvolta nel processo di insegnamento/apprendimento, portatore di una carica affettiva e comunicativa;

- privilegiando un lavoro di collaborazione con i colleghi.

Il clima che si è creato all'interno di ciascun gruppo classe, e la collaborazione tra le classi diverse per la cura del giardino, è favorito dal processo di responsabilizzazione degli studenti sia nell'apprendimento sia nelle attività che li coinvolgono praticamente e in prima persona. La struttura organizzativa è di tipo orizzontale e crea un flusso multilaterale di conoscenze, abilità e informazioni fra gli studenti e tra questi e i docenti, attivando processi continui di feedback.

\section{References}

Bertolini, P. (1996). Voce: Educazione Ambientale. In P. Bertolini, (1996), Dizionario di Pedagogia e Scienze dell'Educazione, Bologna: Zanichelli.

Binanti, L. (2012). Prefazione. In V. De Vitis, (2012), L'educąione ambientale tra biodiversità e intercultura (pp. 11-13). Roma: Anicia

Cambi, F. (2006). Incontro e dialogo. Prospettive della pedagogia interculturale. Roma: Carocci.

Cicirelli, F. (2010). Camilla e il mondo dei giardini. Una fiaba di educarione ambientale. Molfetta: La Meridiana.

De Vitis, V. (2012). L'educazione ambientale tra biodiversità e intercultura. Roma: Anicia.

Frabboni, F. (1989). L'ambiente come laboratorio: quando il territorio si fa aula didattica. Teramo: EIT.

Shiva, V. (2013). Storia dei semi. Milano: Feltrinelli.

Traverso, A. (2012). Educare alla sostenibilità. Ricerca educativa e promozione delle culture. In A. Vischi, (2012), Sviluppo umano e ambiente. Educazione, ricerca, vita buona (pp. 55-72). Milano: EDUCatt. 